\title{
СЦЕНАРИИ ПРОГНОЗА СРОКА СЛУЖБЫ оптического волокна
} в КЛ

\author{
В.Андреев, д.Т.Н., президент ПГУТИ, \\ В.Бурдин, д.Т.н., заведующий кафедрой ЛС и ИТС ПГУТИ, \\ А.Нижгородов, аспирант ПГУТИ / anton.socol2017@yandex.ru
}

УДК 654.1, DOI: 10.22184/2070-8963.2020.89.4.34.43

Рассмотрены возможные сценарии прогноза срока службы оптического кабеля на введенной в эксплуатацию линии связи при использовании рекомендуемой в нормативных документах модели, описывающей взаимосвязь срока службы и вероятности повреждения оптического волокна в кабеле.

Задача прогноза срока службы кабелей телекоммуникационных сетей была актуальна для операторов связи всегда. Это обусловлено тем, что замена кабельных линий является одним из наиболее дорогостоящих этапов реконструкции сети связи, что во многом определяется проблемами доступа к земельным ресурсам, инфраструктуре. Оптические кабели (ОK) не являются исключением. Особенно актуальной эта задача стала сегодня, когда срок службы ОK, введенных в эксплуатацию на сетях связи в 1990-е годы, приблизился и даже превысил декларируемый производителями предельный срок службы 25-30 лет, что соответствует оценкам, полученным на основе теоретических моделей и результатов экспериментальных исследований [1-4].

Известны два основных подхода к прогнозу надежности OK [5]. Предпочтение отдают подходу, базирующемуся на прогнозе срока службы оптического волокна (ОВ) в кабеле $[4,5]$. К настоящему времени методики прогноза срока службы волокна достаточно подробно проработаны, детально описаны и представлены в регламентирующих документах [3, 6-7]. Для прогноза срока службы оптического волокна предлагается следующее выражение [3, 6]:

$$
\mathrm{t}_{\mathrm{a}}=\mathrm{t}_{\mathrm{p}}\left(\frac{\sigma_{\mathrm{p}}}{\sigma_{\mathrm{a}}}\right)^{\mathrm{n}}\left\{\left[1-\frac{\ln (1-\mathrm{F})}{\mathrm{N}_{\mathrm{p}} \mathrm{L}}\right]^{\frac{1}{\mathrm{~m}_{\mathrm{s}}}}-1\right\},
$$

где $\mathrm{t}_{\mathrm{p}}$ - срок службы ОВ;

$\sigma_{\mathrm{a}}$ - нагрузка, прикладываемая к волокну в процессе его срока службы; $\mathrm{L}$ - длина OB, на которую прогнозируют срок службы;

$\mathrm{N}_{\mathrm{p}}$ - число обрывов волокна при прохождении испытаний под нагрузкой (proof-test);

n - параметр прочности кварцевого стекла;

$\mathrm{m}_{\mathrm{s}}$ - параметр распределения Вейбулла; $\mathrm{F}$ - вероятность повреждения ОВ.

Формула (1) может быть записана иначе [6]:

$$
\mathrm{t}_{\mathrm{a}}=\frac{1}{\sigma_{\mathrm{a}}^{\mathrm{n}}}\left\{\left[\frac{\beta^{\mathrm{m}_{\mathrm{s}}}}{\mathrm{L}} \ln \frac{1}{\mathrm{P}}+\left(\sigma_{\mathrm{p}}^{\mathrm{n}} \mathrm{t}_{\mathrm{p}}\right)^{\mathrm{m}_{\mathrm{s}}}\right]^{\frac{1}{\mathrm{~m}_{\mathrm{s}}}}-\sigma_{\mathrm{p}}^{\mathrm{n}} \mathrm{t}_{\mathrm{p}}\right\},
$$

где $\mathrm{P}=1-\mathrm{F}$-вероятность безотказной работы оптического волокна в течение срока службы $\mathrm{t}_{\mathrm{p}}$.

Параметр $\beta$ определяется согласно [6], как:

$$
\beta=\sigma_{p}^{n_{p}} t_{p} N_{p}^{-\frac{1}{m_{s}}}=\sigma_{p}^{n_{p}} t_{p} L_{p}^{\frac{1}{m_{s}}} .
$$

Формула (1) была получена в [8, 9] на основе статической модели разрушения ОВ из плавленого кварцевого стекла $[10,11]$, согласно которой прочность 
кварцевого волокна под действием статической нагрузки изменяется, как:

$$
\mathrm{S}_{\mathrm{i}}^{\mathrm{n}-2}=\mathrm{S}_{\mathrm{i}-1}^{\mathrm{n}-2}-\frac{\sigma_{\mathrm{a}}^{\mathrm{n}} \Delta \mathrm{t}_{\mathrm{i}}}{\mathrm{B}},
$$

где $S_{\mathrm{i}-1}, S_{\mathrm{i}}-$ прочность ОВ в начале и в конце интервала времени $\Delta \mathrm{t}_{\mathrm{i}}$ соответственно; $\sigma_{\mathrm{a}}$ - нагрузка, приложенная к волокну в течение интервала времени $\Delta \mathrm{t}_{\mathrm{i}}$; $\mathrm{B}, \mathrm{n}$ - параметры прочности плавленого кварцевого стекла.

Согласно [8, 9] кумулятивный параметр нагрузки $\sigma_{\mathrm{p}}^{\mathrm{n}} \mathrm{t}_{\mathrm{p}}$ при $\sigma_{\mathrm{p}}^{\mathrm{n}} \mathrm{t}_{\mathrm{p}}$ испытании оптического волокна под нагрузкой (proof-test) может быть также определен, как:

$$
\left(\sigma_{p}^{n} t_{p}\right)=B \cdot S_{0}^{n-2}
$$

где $\mathrm{S}_{0}$ - исходная прочность оптического волокна в начале испытаний.

Следует отметить, что в настоящее время, как правило, используют более корректную двустадийную модель разрушения оптических волокон из кварцевого стекла [12, 13]. Однако, поскольку первая стадия может длиться годами, а вторая протекает за доли секунды, то при анализе срока службы ОВ в кабеле можно ограничиться только первой стадией, воспользовавшись формулой [12]:

$$
\mathrm{S}\left(\mathrm{t}_{\min }\right)^{\mathrm{n}-2}=\mathrm{S}\left(\mathrm{t}_{\max }\right)^{\mathrm{n}-2}+\frac{1}{\mathrm{~B}_{\mathrm{t}_{\min }}} \int_{\max }^{\mathrm{m}_{\max }}\left[\sigma_{\mathrm{a}}(\mathrm{t})\right]^{\mathrm{n}} \mathrm{dt} .
$$

Это справедливо для диапазона изменений $\mathrm{t}$, в котором выполняется условие $\sigma(\mathrm{t}) / \mathrm{S}(\mathrm{t})<\mathrm{r}$.

Здесь $\mathrm{S}\left(\mathrm{t}_{\min }\right), \mathrm{S}\left(\mathrm{t}_{\max }\right)$ - прочность ОВ в начале и в конце интервала времени $\Delta \mathrm{t}=\mathrm{t}_{\max }-$ $\mathrm{t}_{\min }$ в моменты времени $\mathrm{t}_{\min }$ и $\mathrm{t}_{\max }$ соответственно;

r - норма, определяющая переход из первой стадии во вторую;

$\mathrm{B}, \mathrm{n}$ - параметры прочности плавленого кварцевого стекла для первой стадии.

При $\sigma_{\mathrm{a}}(\mathrm{t})=$ const формула (6) принимает вид (4), но должно выполняться условие $\sigma(\mathrm{t}) / \mathrm{S}(\mathrm{t})<\mathrm{r}$. В противном случае следует полагать, что волокно разрушено.

Формулы (1) и (2) с учетом (3), (5) можно привести к следующему виду:

$$
\begin{aligned}
& \ln \frac{1}{\mathrm{P}_{\mathrm{X}}}=\left[(\mathrm{X}+1)^{\mathrm{m}_{\mathrm{s}}}-1\right] \cdot \frac{\mathrm{L}}{\mathrm{L}_{\mathrm{p}}}, \\
& \text { где } \mathrm{X}=\frac{\sigma_{\mathrm{a}}^{\mathrm{n}} \mathrm{t}_{\mathrm{a}}}{\sigma_{\mathrm{p}}^{\mathrm{n}} \mathrm{t}_{\mathrm{p}}} .
\end{aligned}
$$

Из (7), (8) следует, что, если известны параметры прочности кварцевого стекла В, $\mathrm{n}$ и параметр распределения Вейбулла $\mathrm{m}_{\mathrm{s}}$, которые приводятся как справочные данные в рекомендациях $[4,6]$, то для того чтобы оценить вероятность безотказной работы ОВ в течение заданного срока службы необходимо и достаточно знать кумулятивный параметр нагрузки в прошлом $\sigma_{\mathrm{p}}^{\mathrm{n}} \mathrm{t}_{\mathrm{p}}$ и кумулятивный параметр нагрузки в будущем $\sigma_{\mathrm{a}}^{\mathrm{n}} \mathrm{t}_{\mathrm{a}}$. При этом полагается, что прикладываемые к волокну как в прошлом, так и в будущем нагрузки - величины постоянные.

Очевидно, что с точки зрения прогноза срока службы ОВ в кабеле, введенном в эксплуатацию, данное допущение некорректно. Это наглядно демонстрирует приведенная на рис.1 широко известная специалистам иллюстрация нагрузок на волокно и изменений прочности кварцевых ОВ в кабеле в процессе изготовления последнего, строительства и эксплуатации кабельной линии [14].

Можно предположить, что рассматриваемый срок службы оптического кабеля может быть разбит на интервалы времени, в течение которых выполняется только один технологический процесс, характеризующийся временем исполнения и приложенной к ОВ в кабеле нагрузкой, не изменяющейся во время выполнения данного технологического процесса. Такой подход позволил ввести выражение для эквивалентного значения напряжения в ОВ кабеля [15]:

$$
\sigma_{\text {eq }}^{n}=\frac{1}{\mathrm{t}_{\mathrm{s}}}\left[\sum_{\mathrm{i}=1}^{\mathrm{M}_{\mathrm{t}}}\left(\sigma_{\mathrm{i}}^{\mathrm{n}} \mathrm{t}_{\mathrm{i}}\right)^{\mathrm{m}_{\mathrm{s}}} \frac{\mathrm{L}_{\mathrm{i}}}{\mathrm{L}_{\mathrm{s}}}\right]^{\frac{1}{\mathrm{~m}_{\mathrm{s}}}},
$$

где $\mathrm{M}_{\mathrm{t}}$ - количество интервалов времени, на которые разбивается срок службы оптического кабеля;

$\sigma_{\text {eq }}$-эквивалентное значение нагрузки на оптическое волокно в кабеле в течение срока службы;

$\mathrm{t}_{\mathrm{i}}, \mathrm{L}_{\mathrm{i}}, \sigma_{\mathrm{i}},-$ - значения длительности і-того интервала времени, длины кабеля и остаточного напряжения на волокне для этого интервала времени;

$\mathrm{t}_{\mathrm{s}}, \mathrm{L}_{\mathrm{s}}$ - значения длительности срока службы и длины кабеля, для которых определяется эквивалентное напряжение в волокне.

К надежности кабельных линий связи предъявляются весьма высокие требования. Согласно [16] магистральные кабельные линии связи должны соответствовать следующим основным 


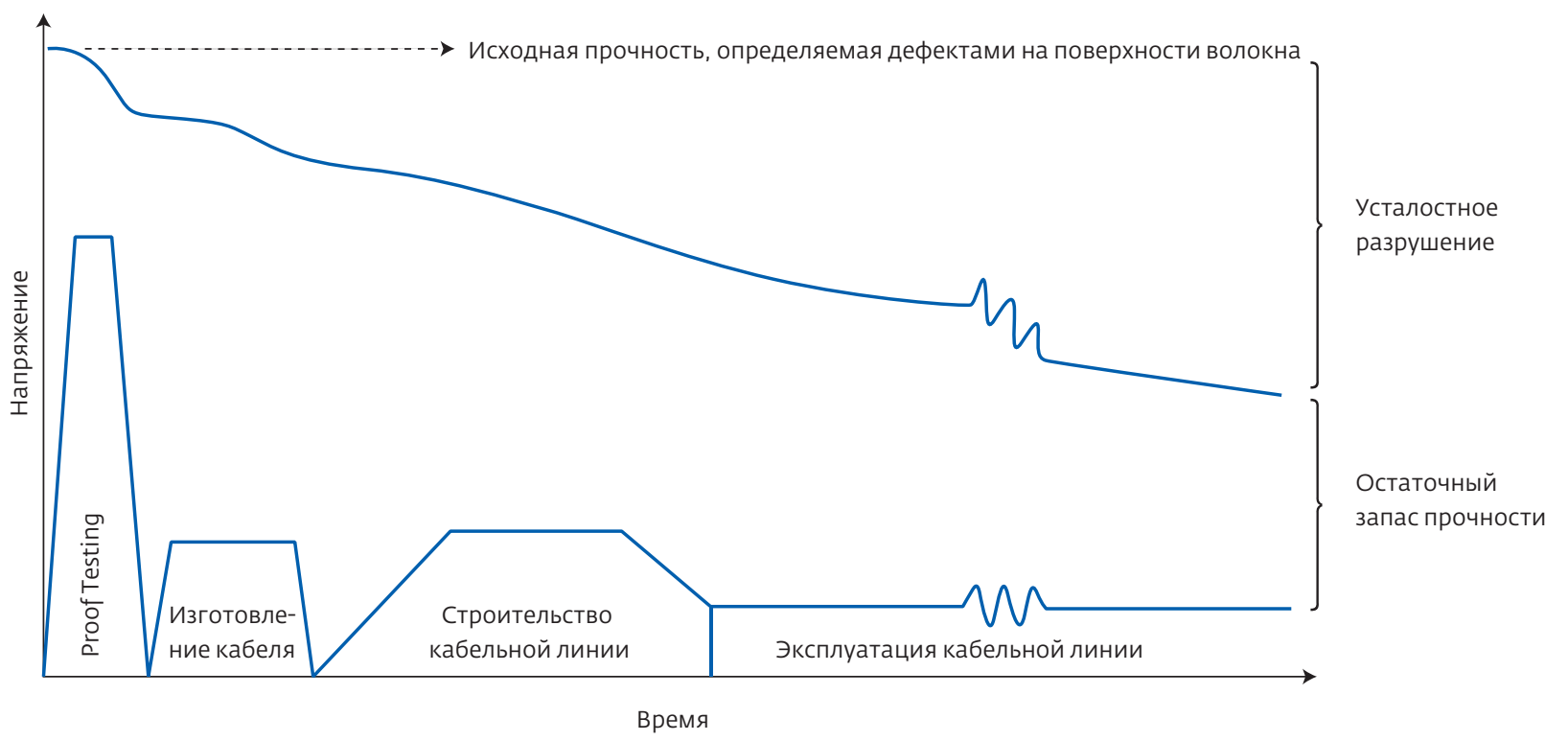

Рис.1. Иллюстрация изменений нагрузок на кварцевое оптическое волокно в кабеле и его прочности в процессе изготовления кабеля, строительства и эксплуатации линии связи. Источник: [14]

нормативным показателям по надежности их работы:

- наработка на отказ на 100 км линии передачи: не ниже $\mathrm{T}_{\mathrm{o}}=34375$ ч;

- коэффициент простоя: не выше $\mathrm{K}_{\Pi}=2,55 \cdot 10^{-4}$;

- коэффициент готовности: не ниже Кг = 0,99970 .

Однако, уже при $\mathrm{K}_{\Gamma}>0,9$ в (7) можно полагать X"1, откуда следует, что приближенно

$$
\ln \frac{1}{\mathrm{P}_{\mathrm{X}}}=\mathrm{m}_{\mathrm{s}} \mathrm{X} \cdot \frac{\mathrm{L}}{\mathrm{L}_{\mathrm{p}}} .
$$

С учетом данного приближения формулу для эквивалентного остаточного напряжения в волокне можно записать как:

$$
\sigma_{\text {eq }}^{n}=\frac{1}{m_{s} L_{s} t_{s}} \sum_{i=1}^{M_{t}} L_{i} \sigma_{i}^{n} t_{i}
$$

Поскольку в процессе строительства и эксплуатации кабельной линии отдельные участки OK, а, соответственно, и волокна в нем, могут оказаться под действием неодинаковой нагрузки, то формулу (9) целесообразно привести к виду:

$$
\sigma_{\text {eq }}^{n}=\frac{1}{m_{s} L_{s} t_{s}} \sum_{i=1}^{M_{t}} \sum_{j=1}^{M_{L}} L_{i j} \sigma_{i j}^{n} t_{i},
$$

где $\mathrm{M}_{\mathrm{L}}$ - количество участков, на которые разбивается длина кабеля на і-том интервале времени.

Из (12) следует выражение для кумулятивного параметра напряжения в волокне:

$$
\left(\sigma_{\mathrm{a}}^{\mathrm{n}} \mathrm{t}_{\mathrm{a}}\right)_{\mathrm{eq}}=\left[\sum_{\mathrm{i}=1}^{\mathrm{M}_{\mathrm{t}}} \sum_{\mathrm{j}=1}^{\mathrm{M}_{\mathrm{L}}}\left(\sigma_{\mathrm{ij}}^{\mathrm{n}_{\mathrm{i}}}\right)^{\mathrm{m}_{\mathrm{s}}} \frac{\mathrm{L}_{\mathrm{ij}}}{\mathrm{L}_{\mathrm{s}}}\right]^{\frac{1}{\mathrm{~m}_{\mathrm{s}}}} .
$$

Поскольку эквивалентные значения напряжения в волокне и его кумулятивного параметра уже приведены к длине кабеля, для которой осуществляется прогноз, то формула (7) принимает следующий вид:

$$
\ln \frac{1}{\mathrm{P}_{\mathrm{X}}}=\left[\left(\mathrm{X}_{\mathrm{eq}}+1\right)^{\mathrm{m}_{\mathrm{s}}}-1\right]
$$

При этом, с учетом (6) и (8), величина $\mathrm{X}_{\mathrm{eq}}$ в (12) определяется, как:

$$
\mathrm{X}_{\mathrm{eq}}=\frac{\left(\sigma_{\mathrm{a}}^{\mathrm{n}} \mathrm{t}_{\mathrm{a}}\right)_{\text {eq }}}{\sigma_{0}^{\mathrm{n}} \mathrm{t}_{0}-\left(\sigma_{\mathrm{p}}^{\mathrm{n}} \mathrm{t}_{\mathrm{p}}\right)_{\text {eq }}},
$$

где $\mathrm{t}_{0}, \sigma_{0}$ - параметры испытания оптического волокна под напряжением (proof-test).

Кумулятивный параметр данного теста может оцениваться по выражению (5). Эквивалентные 
значения параметров $\mathrm{t}_{\mathrm{p}} \sigma_{\mathrm{p}}^{\mathrm{n}}$ и $\left(\mathrm{t}_{\mathrm{a}} \sigma_{\mathrm{a}}^{\mathrm{n}}\right)_{\mathrm{eq}}$ в (15) рассчитываются по формуле (13) для процессов, протекавших в прошлом и ожидаемых в будущем соответственно.

Естественно, для расчета данных величин необходимо знать характеристики процессов: продолжительность и нагрузку на волокно в течение процесса. Эти характеристики определяют в результате анализа технологии производства кабеля, сбора статистических данных [15]. Конечно же, при производстве кабеля и строительстве линий связи стремятся соблюдать технологическую дисциплину, однако отклонений от нормы полностью исключить нельзя.

В меньшей степени эти отклонения характерны для производства ОК, в большей - для процессов строительства и эксплуатации кабельных линий. В частности, при работах, связанных с перемоткой кабеля, по прокладке кабеля и др. Поскольку за соблюдением технологии осуществляется $\mathrm{koH}^{-}$ троль, то эти отклонения, как правило, носят кратковременный характер, но прилагаемые при этом к волокну нагрузки могут быть весьма значительными. Таким образом, в общем случае параметр $\mathrm{X}_{\text {eq }}$ в (14) нельзя полагать детерминированным. Как следствие, и рассчитываемая по формуле (14) величина $\mathrm{P}_{\mathrm{X}}$ также есть величина случайная. Тогда в общем случае искомая вероятность безотказной работы определяется интегралом:

$$
\mathrm{P}_{\mathrm{f}}=\int_{\mathrm{X}_{\min }}^{\infty} \mathrm{P}_{\mathrm{p}}(\mathrm{X}) \cdot \mathrm{P}_{\mathrm{X}}(\mathrm{X}) \mathrm{dX},
$$

где $\mathrm{X}_{\min }{ }^{-}$минимальное значение аргумента $\mathrm{X}$;

$\mathrm{P}_{\mathrm{p}}(\mathrm{X})$ - вероятность того, что аргумент примет значение X.

Рассмотрим случайную величину $\mathrm{X}_{\text {еq }}$ подробнее. Как уже было отмечено, существенные отклонения характеристик технологических процессов от их среднестатистических значений являются редкими и кратковременными событиями. Учитывая это, а также то, что при прогнозах срока службы мы ориентируемся на "наихудший случай", отклонениями в меньшую сторону будем пренебрегать, полагая, что минимальное значение случайной величины $\mathrm{X}_{\text {eq }}$ определяется среднестатистическими характеристиками технологических процессов. При этом эта случайная величина изменяется от своего минимального значения до бесконечности. С учетом вышесказанного можно допустить, что рассматриваемая случайная величина описывается законом Парето [17]:

$$
\mathrm{P}_{\mathrm{p}}(\mathrm{X})=\frac{\mathrm{a}}{\mathrm{X}_{\min }}\left(\frac{\mathrm{X}_{\min }}{\mathrm{X}}\right)^{\mathrm{a}+1},
$$

где а - параметр распределения Парето.

Подставляя (17) в (16) и сделав замену переменной

$$
\mathrm{y}=\mathrm{X} / \mathrm{X}_{\min }
$$

получаем:

$$
P_{f}=a \int_{1}^{\infty} P\left(y \cdot x_{\min }\right) \frac{d y}{y^{a+1}} .
$$

Результат интегрирования (20) находим численными методами.

Таким образом, вероятность безотказной работы предлагается рассчитывать следующим образом. Предварительно, используя среднестатистические характеристики технологических процессов, для заданного момента времени по формулам (13), (15) рассчитываем минимальное значение параметра $\mathrm{X}_{\min }=\mathrm{X}_{\mathrm{eq}}$. После чего для найденного значения рассчитываем вероятность безотказной работы. Если мы пренебрегаем случайным характером характеристик технологических процессов, то расчет выполняем по формуле (14), подставляя в нее параметр $\mathrm{X}_{\min }=\mathrm{X}_{\mathrm{eq}}$ и полагая $\mathrm{P}_{\mathrm{f}}=\mathrm{P}_{\mathrm{X}}$. Если же случайный характер характеристик технологических процессов учитывается, то расчет осуществляем

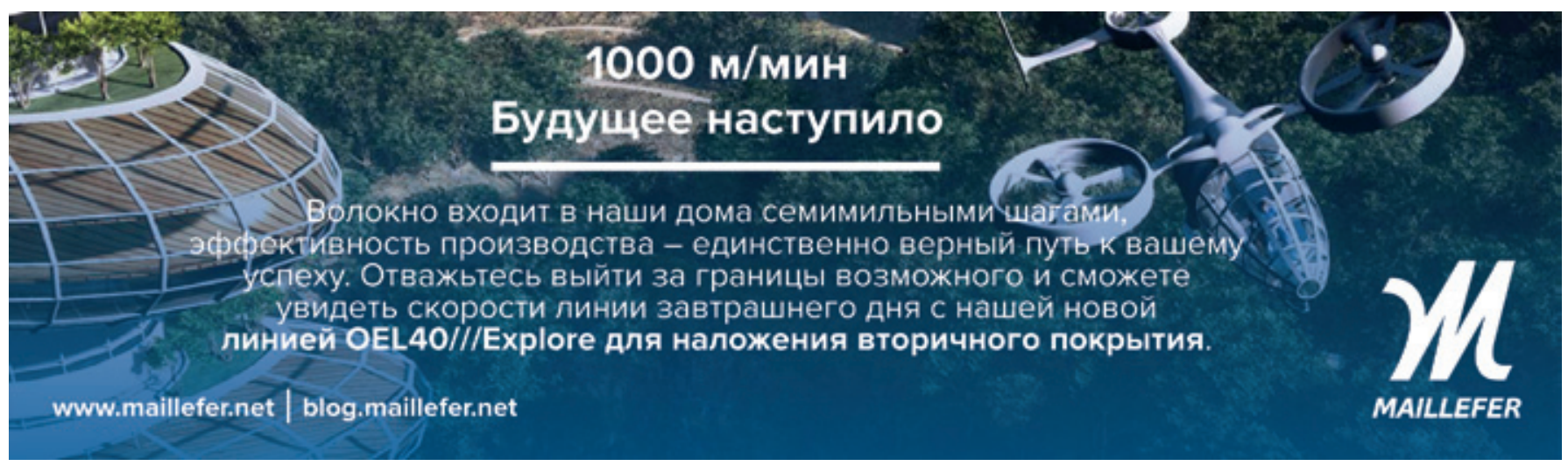




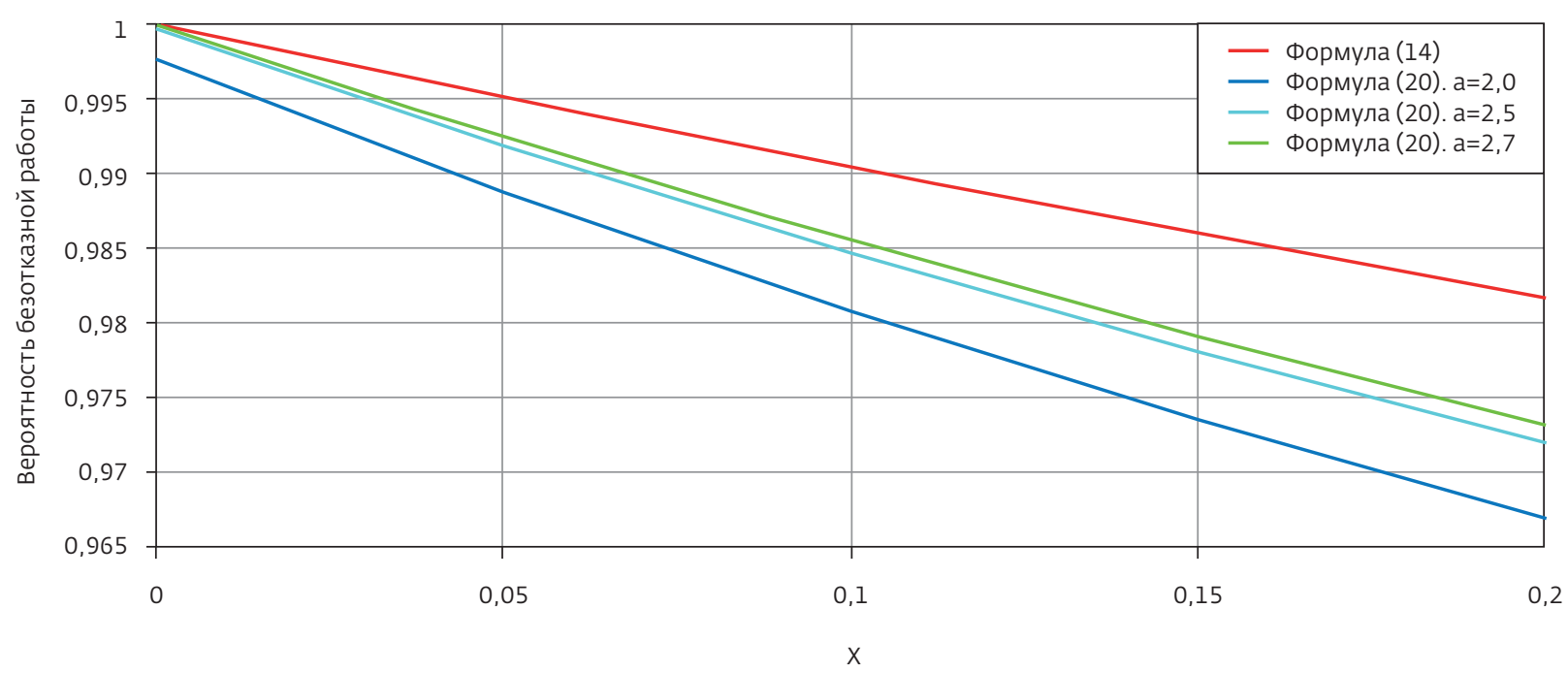

Рис.2. Зависимости вероятности безотказной работы от параметра $\mathrm{X}_{\text {eq }}$

по формуле (20), подставляя в нее значение параметра $\mathrm{X}_{\min }=\mathrm{X}_{\mathrm{eq}}$. Для определения срока службы описан ным выше способом рассчитываются зависимости вероятности безотказной работы от срока службы, по которым находим искомые оценки.

На рис.2 представлены зависимости вероятности безотказной работы от параметра $\mathrm{X}_{\text {еq }}$, вычисленные по формуле (12) и по формуле (20) при $\mathrm{X}_{\mathrm{min}}=\mathrm{X}_{\mathrm{eq}}$. Как видим, даже при достаточно больших значениях параметра зависимости близки к линейным. При этом наклон кривых, рассчитанных по формуле (20), сильно зависит от значения параметра Парето.

Безусловно, если базироваться только на среднестатистических данных о технологических процессах производства оптических кабелей, строительства и эксплуатации ВОЛс, описанный выше способ даст весьма грубые оценки искомой вероятности безотказной работы, что обусловлено существенным отличием данных для реальных кабельных линий от среднестатистических для заданных типов ОК и методов их прокладки. Во многом это связано с воздействием внешних факторов, включая человеческий фактор, при строительстве и эксплуатации кабельных линий, с деградацией элементов конструкции кабеля, что влияет на прикладываемые к оптическим волокнам нагрузки [4, 18-19]. Неслучайно, что при прогнозах срока службы кабелей, введенных в эксплуатацию, рекомендуется опираться на всю совокупность данных о кабельной линии, включая статистику повреждений, данные мониторинга и результаты специальных измерений $[20,21]$.

Важнейшую роль здесь играют специальные измерения, предназначенные для выявления "плохих" участков кабеля, на которых его оптические волокна подвержены ускоренной деградации. И в первую очередь, к таким измерениям относятся измерения бриллюэновским оптическим рефлектометром (BOTDR - Brillouin optical time domain reflectometer), работающим во временной области [22, 23]. Данное метрологическое средство предназначено для измерений распределений остаточных растягивающих напряжений в ОВ вдоль его длины [22-26]. Участки волокна с ускоренной деградацией выявляются по превышению приложенными к волокну остаточными растягивающими напряжениями допустимых пороговых значений [22-27].

В силу принципов работы BOTDR его возможности по выявлению напряжений, связанных с радиальными воздействиями, изгибами и скручиванием волокон, ограничены. Так, в работе [28] показано, что уверенно с помощью BOTDR можно выявлять изгибы ОВ с радиусом изгиба менее 25 мм. Однако это значение меньше минимально допустимого значения радиуса изгиба [27]. Вместе с тем напряжения, создаваемые на изгибах OB, не менее опасны с точки зрения деградации волокон в кабеле, чем растягивающие нагрузки $[7,18,27]$. По этой причине рекомендуется измерения остаточных растягивающих напряжений в ОВ, осуществляемые с помощью 
BOTDR, дополнять оценкой распределений радиусов изгиба волокон вдоль кабеля [29]. Методы измерений распределений кривизны волокон вдоль кабеля известны [29-35], однако специализированных полевых средств измерений пока, к сожалению, нет.

Вторая и, вероятно, наиболее существенная проблема заключается в том, что упомянутые средства измерений по своему назначению и принципам работы позволяют оценивать остаточные напряжения в волокне, но не его состояние, прочность. Методы и средства неразрушающего контроля OB в кабеле на линии связи, введенной в эксплуатацию, пока не разработаны. Если обратиться к формулам (7), (8) для прогноза срока службы волокна в кабеле, то получаем, что для расчета параметра X в (8) числитель по результатам специальных измерений нам известен, а знаменатель - нет. Как следствие, приходится производить отсчет от исходной прочности оптического волокна, оценивая ее по результатам испытаний последнего под нагрузкой (proof test). И здесь, при расчете вероятности безотказной работы в течение заданного срока службы по результатам измерений остаточных напряжений в ОВ, возможны разные сценарии. Рассмотрим их подробнее на следующем примере.

Пусть в некоторый момент времени в течение эксплуатации кабельной линии выполнено тестирование и получена оценка остаточного напряжения на оптическом волокне $\sigma_{\mathrm{a}}$ и необходимо оценить вероятность безотказной работы в течение срока службы $t_{a}$, начиная с момента выполнения измерений. Исходную прочность, как было уже отмечено выше, будем оценивать кумулятивным параметром нагрузки $\sigma_{\mathrm{p}}^{\mathrm{n}} \mathrm{t}_{\mathrm{p}}$ испытания ОВ под напряжением (proof test). И пусть при этом с момента завершения данного теста до момента выполнения измерений прошло время $\mathrm{t}_{\mathrm{L}}$.

Если статистические данные о технологических процессах в прошлом для рассматриваемой кабельной линии отсутствуют, можно предположить, что нагрузка, оценка которой получена в результате измерений, была приложена к волокну в течение всего срока службы кабеля и будет приложена к нему в будущем. Тогда возможны два варианта расчета эквивалентного параметра $\mathrm{X}_{\mathrm{eq}}$ В первом случае по формуле (8) как:

$$
\mathrm{X}=\frac{\sigma_{\mathrm{a}}^{\mathrm{n}}\left(\mathrm{t}_{\mathrm{L}}+\mathrm{t}_{\mathrm{a}}\right)}{\sigma_{0}^{\mathrm{n}} \mathrm{t}_{0}} .
$$

Во втором случае расчет выполняется по формуле (13) следующим образом:

$$
\mathrm{X}=\frac{\sigma_{\mathrm{a}}^{\mathrm{n}} \mathrm{t}_{\mathrm{a}}}{\sigma_{0}^{\mathrm{n}} \mathrm{t}_{0}-\sigma_{\mathrm{a}}^{\mathrm{n}} \mathrm{t}_{\mathrm{L}}} .
$$

Если статистические данные о технологических процессах в прошлом для рассматриваемой кабельной линии известны, то можно приближенно учесть "историю" службы ОВ. В этом случае вычисления выполняем по формуле (15), записанной следующим образом:

$$
\mathrm{X}_{\mathrm{eq}}=\frac{\sigma_{\mathrm{a}}^{\mathrm{n}} \mathrm{t}_{\mathrm{a}}}{\sigma_{\mathrm{o}}^{\mathrm{n}} \mathrm{t}_{\mathrm{o}}-\left(\sigma_{\mathrm{p}}^{\mathrm{n}} \mathrm{t}_{\mathrm{p}}\right)_{\mathrm{eq}}} .
$$

Подставляя (21)-(23) в (14) или (20), получаем оценки вероятности безотказной работы для заданного срока службы оптического волокна. Если случайным характером технологических процессов

\begin{tabular}{|c|c|c|c|}
\hline $\begin{array}{l}\text { №№ } \\
\text { п.п. }\end{array}$ & Процесс эксплуатации & Длительность, ч & Напряжение, \% \\
\hline 1 & $\begin{array}{l}\text { Среднее значение остаточного напряжения, приложенного } \\
\text { ежедневно }\end{array}$ & 78840 & 0,08 \\
\hline 2 & $\begin{array}{l}\text { Дополнительное напряжение при гололедной и ветровой } \\
\text { нагрузке }\end{array}$ & 72 & 0,20 \\
\hline 3 & $\begin{array}{l}\text { Дополнительное напряжение при низкой отрицательной } \\
\text { температуре }\end{array}$ & 72 & 0,12 \\
\hline 4 & Дополнительное максимальное напряжение при порывах ветра & $<1$ & 0,11 \\
\hline 5 & $\begin{array}{l}\text { Дополнительное напряжение при минимальной температуре } \\
\left.\text { (ниже }-27^{\circ} \mathrm{C}\right)\end{array}$ & $2-3$ & 0,12 \\
\hline
\end{tabular}
пренебрегаем, то используем формулу (14), если нет,

Таблица 1. Оценки остаточных напряжений в ОВ в кабеле и интервалы времени 
Таблица 2. Вклад в деградацию ОВ процессов производства кабеля

\begin{tabular}{|c|l|c|c|c|}
\hline $\begin{array}{c}\text { №№ } \\
\text { п.п. }\end{array}$ & $\begin{array}{c}\text { Наименование технологического } \\
\text { процесса производства кабеля }\end{array}$ & Длина, км & Длительность, мин & Напряжение, \% \\
\hline 1 & Окраска волокна & 25 & 41,7 & $7,69 \cdot 10^{-7}$ \\
\hline 2 & Изгововление модуля & 4 & 10 & $3,21 \cdot 10^{-6}$ \\
\hline 3 & Скрутка модулей в сердечник & 4 & 20 & 0,064 \\
\hline 4 & Наложение внешних покровов & 4 & 40 & $9,35 \cdot 10^{-14}$ \\
\hline
\end{tabular}

то выражение (20). Рассчитав зависимости вероятности безотказной работы от срока службы, получаем оценки срока службы ОВ с заданной вероятностью безотказной работы.

Для сравнения получаемых по разным сценариям оценок выполним вычисления для примера, достаточно подробные исходные данные для которого содержатся в работах [36-37]. В них представлены данные для линии с кабелем типа OPT-GW, включающим 12 стандартных одномодовых оптических волокон, который был произведен в 1986 году и уже в первом квартале 1986 проложен на линии связи. Около 3,7 км кабеля было подвешено на высоковольтной линии 138 кВ. Через девять лет, в 1995 году, по решению оператора связи кабель был демонтирован и с согласия производителя кабеля и поставщика волокна подвергнут тестированию.

Результаты испытаний показали, что за девять лет эксплуатации волокна не деградировали. При этом в [36-37] приведены оценки остаточных напряжений в оптических волокнах в кабеле и интервалов времени, в течение которых эти напряжения были приложены. Данные представлены в табл.1.

Внешний диаметр упомянутого ОК равен 12 мм. Для отечественных аналогов данного кабеля (типа ОКГТ - встроенного в грозотрос) с таким же диаметром разрывная прочность составляет $87 \mathrm{kH}$ [38]. Согласно принятым нормам для кабелей такого типа допускается максимальное растягивающее напряжение до 70\% от максимальной прочности на разрыв [39, 40]. Учитывая особенности трансфера нагрузки от кабеля к оптическим волокнам и полагая, что тяговые усилия при подвеске не превышали 60\% от максимальной прочности на разрыв, будем полагать, что прикладываемая при подвеске кабеля к оптическим волокнам нагрузка составляет 0,32\%.

Согласно принятым нормам [41] на подвеску гро зотроса длиной 5 км отводится 5,41 смены. Одна смена длится 8 ч. При этом собственно раскатка грозотроса занимает 25\% от всего отведенного на его подвеску времени. Таким образом, подвеска одной строительной длины оптического кабеля в грозо тросе (OPT-GW) длиной в 5 км составляет около 11 ч. В целях упрощения, при учете процессов строительства кабельной линии ограничимся подвеской кабеля, полагая, что именно этот технологический процесс вносит основной вклад в остаточные напряжения в волокнах ОK.

Чтобы учесть вклад в деградацию ОВ процессов производства кабеля воспользуемся данными, представленными в работе [15]. Данные сведены в табл.2. Как следует из таблицы, основной вклад в остаточные напряжения в волокне при производстве вносит скрутка модулей в сердечник. Это позволяет в целях упрощения ограничиться здесь учетом технологических процессов, представленных в табл.2, пренебрегая последующими операциями изготовления кабеля, встроенного в грозотрос.

Будем считать, что тестирование выполнено через девять лет после ввода кабеля в эксплуатацию. И пусть по результатам измерений получена оценка остаточного напряжения в оптическом волокне $\sigma_{\mathrm{a}}=0,1 \%$. Для вышеуказанных условий выполним расчеты по формулам (14) и (20) для рассмотренных сценариев. Результаты вычислений приведены на рис. 3 и 4.

Как следует из этих графиков, разные сце нарии дают разные оценки, хотя и примерно 


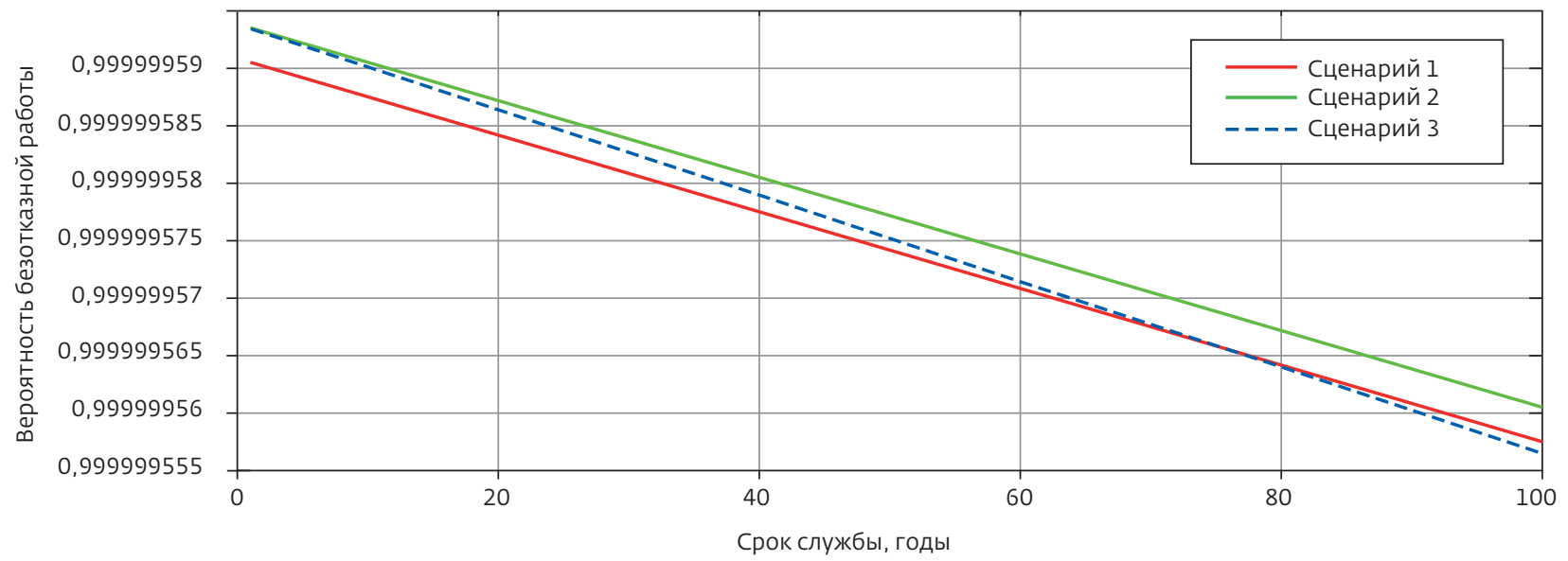

Рис.3. Результаты вычислений по формуле (14). Случайным характером технологических процессов пренебрегаем

одного порядка. При этом учет случайного характера технологических процессов дает увеличение оценок вероятности повреждения кабеля более чем на порядок. Хотя, следует отметить, что для рассматриваемого примера во всех случаях срок службы с требуемыми значениями вероятности безотказной работы превышает 25 лет. Это согласуется с результатами обследования образца кабеля после девяти лет эксплуатации, представленными для данного примера в [36-37].

\section{ЗАКЛЮЧЕНИЕ}

Представленный выше анализ, на взгляд авторов, позволяет сделать следующие выводы.

Для повышения точности прогнозов срока службы OK, введенного в эксплуатацию, необходимо учитывать всю совокупность данных о кабеле и линии

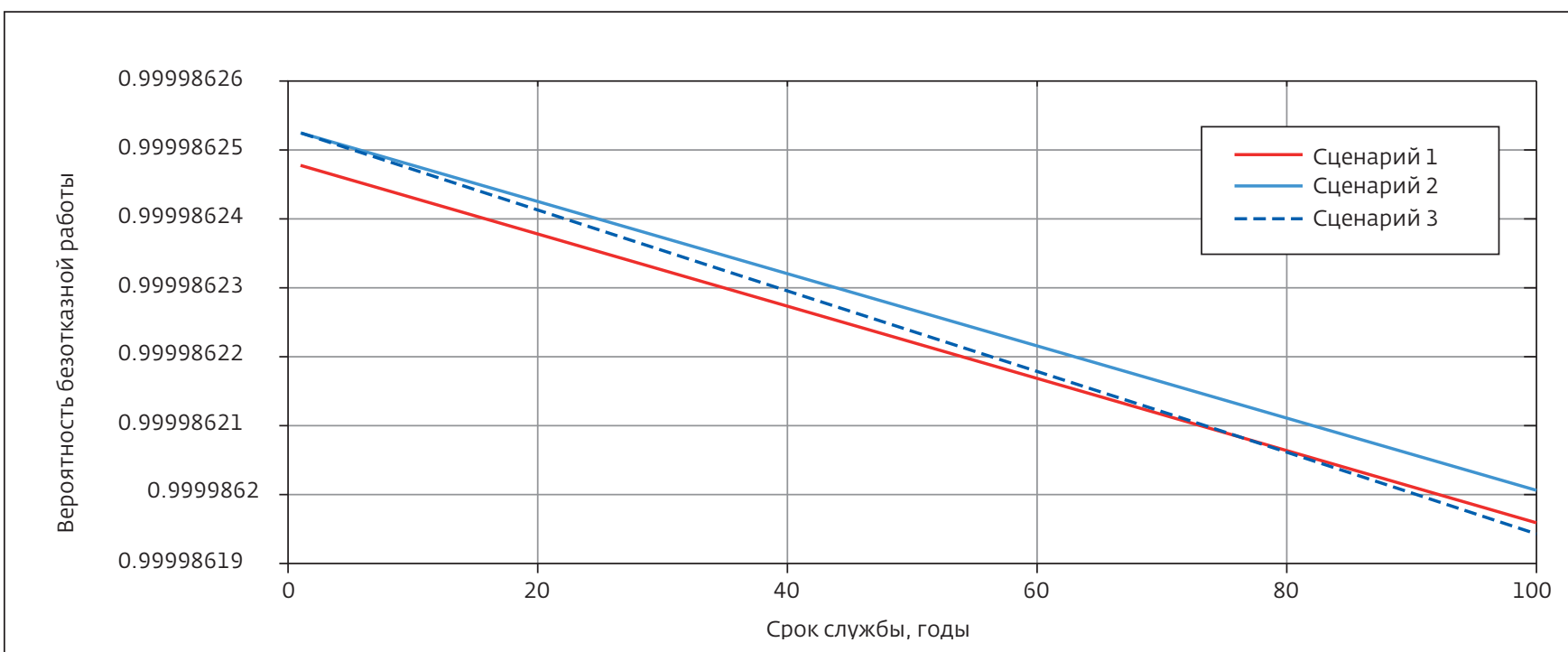

Рис.4. Результаты вычислений по формуле (20). Предполагается, что параметры технологических процессов носят случайный характер, параметр Парето $a=2,7$ 
связи, включая статистические характеристики технологических процессов изготовления кабеля, строительства и технической эксплуатации кабельной линии, статистику повреждений кабеля, дан ные мониторинга и результаты измерений оценок остаточных напряжений в оптических волокнах и оценок их состояния.

Предложенное решение для учета случайного характера технологических процессов изготовления ОК, строительства и технической эксплуатации кабельной линии позволяет более корректно оценивать срок службы и вероятность безотказной работы оптического кабеля, введенного в эксплуатацию. Однако оно требует сбора статистических данных для отдельных типов ОК и способов их прокладки, включая характеристики трансфера нагрузки от кабеля к волокну. Кроме того, требуется разработка методики определения параметра Парето и собственно его определение для конкретных условий.

Для повышения достоверности прогноза срока службы ОК на линии, введенной в эксплуатацию, нужны измерения распределений растягивающих напряжений в оптических волокнах и распределений радиусов изгиба (кривизны) ОВ вдоль кабеля. Для этого необходимы соответствующие полевые приборы и, следовательно, их разработка.

И наконец, для точных прогнозов срока службы OK на линии, введенной в эксплуатацию, необходимы оценки состояния ОВ в кабеле. А это, в свою очередь, требует проведения поисковых научноисследовательских работ и разработки методов и средств неразрушающего контроля оптических волокон в кабеле непосредственно на линии связи.

\section{ЛИТЕРАТУРА}

1. Воронцов А.С., Гурин О.И., Мифтяхетдинов С.Х., Никольский К.К., Питерских С.Э. Оптические кабели связи российского произ водства. Справочник. - М.: Эко-Трендз, 2003. 283 c.

2. Van Vickle P. Optical Fiber Cable Design \&Reliability // IEEE 802.3 NG-EPON Meeting. May 2014. Norfolk. VA. http://www.ieee802.org/3/ad_hoc/ ngepon/public/ may14/vanvickle_ngepon_01_0514. pdf.

3. ITU-T G-series Recommendations - Supplement 59, SERIES G: TRANSMISSION SYSTEMS AND MEDIA, DIGITAL SYSTEMS AND NETWORKS, Guidance on optical fibre and cable reliability, (02/2018).21p.

4. Цым А.Ю. Сроки службы оптических кабелей. Анализы. Риски // IV Всероссийская научнотехническая конференция "Линии связи XXI века": сб. тезисов. - Самара, 2019.
5. Ларин Ю.Т. Сравнительный анализ двух подходов к надежности оптических кабелей // Наука и техника. 2009. № 2(315). С. 3-7.

6. IEC TR 62048:2014 Optical fibres - Reliability - Power law theory. 2014. 66 p.

7. Glaesemann G.S. Optical Fiber Mechanical Reliability. Review of Research at Corning's Optical Fiber Strength Laboratory. White Paper, WP 8002. - Corning Inc., July 2017. 62 p.

8. Mitsunaga Y., Katsuyama Y., Ishida Y. Reliability assurance for long-length optical fibre based on proof testing // Electronics Letters. 1981. V. 17. No. 16. P. 567-568.

9. Mitsunaga Y., Katsuyama Y., Kobayashi H., Ishida Y. Failure prediction for long length optical fiber based on proof testing // Journal of Applied Physics. 1982. V. 53. No. 7. P. 4847-4853.

10. Evans A.G., Wiederhorn S.M. Proof testing of $\mathrm{ce}^{-}$ ramic materials an analytical basis for failure prediction // International Journal of Fracture. 1974. V. 10. No. 3. P. 379-392. DOI: 10.1007/bf00035499.

11. Ritter J.E., Jakus K. Applicability of Crack Velocity Data to Lifetime Predictions for Fused Silica Fibers // Journal of the American Ceramic Society. 1977. V. 60(3-4). P. 171-171. DOI: 10.1111/j.1151 2916.1977.tb15500.x.

12. Hanson T.A., Glaesemann G.S. Incorporation multi-region crack growth into mechanical reliability predictions for optical fiber // Materials Science. 1997. V. 32. P. 5305-5311.

13. Semjonov S., Glaesemann G.S. High-Speed Tensile Testing of Optical Fibers - New Understanding for Reliability Prediction. In: E.Suhir, Y.C.Lee, C.P.Wong (eds) Micro- and Opto-Electronic Materials and Structures: Physics, Mechanics, Design, Reliability, Packaging. - Boston, MA: Springer, 2007. P. A595-A625.

14. Castilone R.J., Glaesemann G.S., Hanson T.A. Extrinsic Strength Measurements and Associated Mechanical Reliability Modeling of Optical Fiber // 16th Annual National Fiber Optics Engineers Conference. - Denver, CO, 2000. P. 1-9.

15. Da Silva A.C., Hirose F.N., Neto J.A.M., Furtado J.M.I. Optimization of Loose Tube Optical Cable Manufacture Process Based on Optical Fiber $\mathrm{Me}^{-}$ chanical Behavior // Proceedings of the 50th IWCS. 2001. P. 249-252.

16. ВСН 116-93. Инструкция по проектированию линейно-кабельных сооружений связи. - M.: Минсвязи России, 1993. 29 с.

17. Mandelbrot B. The Fractal Geometry of $\mathrm{Na}^{-}$ ture. - M.: Institute of Computer Science, 2002. $656 \mathrm{p}$. 
18. Овчинникова И. Определение надежности оптических кабелей // Технологии и средства связи. 2009. № 3. С. 39-41.

19. Корякин А.Г., Овчинникова И.А. Влияние внешних факторов на надежность оптических кабелей // Вестник МЭИ. 2011. № 3. С. 52-56.

20. Veitenheimer T., Cano J., Dorn Ch. Predictive Fiber Monitoring for Aging Optical Networks // NTest WP. 2011. 4 p. http://www.ntestinc.com/papers/fibermonitoring.pdf (11.04.20).

21. Koga H., Kuwabara T., Mitsunaga Y. Future maintenance systems for optical fiber cables // ICC-91 Proceedings. 1991. P. 0323-0329.

22. Kurashima T., Horiguchi T., Izumita H., Furukawa S.I., Koyamada Y. Brillouin optical-fiber time domain reflectometry // IEICE Transactions on Communications. 1993. V. E76-B(4). P. 382-390.

23. BOTDR Measurement Techniques and Brillouin Backscatter Characteristics of Corning Single-Mode Optical Fibers // Corning WP 4259. 2015. 9 p. https:// www.corning.com/media/worldwide/coc/documents/Fiber/RC-\%20White\%20Papers/WP-General/ WP 4259_01-15.pdf (11.04.20).

24. Horiguchi T., Kurashima T., Tateda M. Tensile strain dependence of Brillouin frequency shift in silica optical fibers // IEEE Photonics Technology Letters. 1989. V. 1. No. 5. P. 107-108. DOI: $10.1109 / 68.34756$.

25. Акопов С.Г. Контроль бриллюэновским рефлектометром технологии производства оптических кабелей // Вестник связи. 2003. № 4. С. 136138.

26. Корн В.М., Длютров О.В., Авдеев Б.В., Барышников Е.Н. О применении метода Мандельштам-Бриллюэновского рассеяния для измерения характеристик оптических кабелей // Кабели и провода. 2004. № 5 (288). С. 19-21.

27. Castilone R.J. Mechanical Reliability: Applied Stress Design Guidelines // Corning WP 5053. 2001. 4 p. https://www.corning.com/media/worldwide/ coc/documents/Fiber/RC-\%20White\%20Papers/WPGeneral/WP 5053_07-01.pdf (11.04.20).

28. Богачков И.В. Исследование влияния изгибов оптических волокон различных типов на бриллюэновские рефлектограммы // T-Comm: Телекоммуникации и транспорт. 2019. Т. 13. № 3. C. 75-79.

29. Sankawa I., Koyamada Y., Furukawa S.I., Horiguchi T., Tomita N., Wakui Y. Optical fiber line surveillance system for preventive maintenance based on fiber strain and loss monitoring // IEICE Transactions on Communications. 1993. V. E76B(4). P. 402-409.
30. Anderson D.R., Johnson L., Bell F.G. Troubleshooting optical-fiber networks. Understanding and using your optical time-domain reflectometer. - Elsevier, 2004.

31. Chen H., Chen X., Yao X.S. Distributed fiber bend and stress measurement for determining optical fiber reliability by multi-wavelength optical reflectometry // Patent US 2014/0362367, 2014.

32. Okamoto K., Nakamura A., Koshikiya Y., Watanabe H., Manabe T. Highly Sensitive Monitoring of Progressive Microbending Loss Using 1-mmband Mode-detection OTDR // Proceedings of the 65th IWCS Conference. 2016. P. 228-233.

33. Burdin V.A. Methods of optical fiber curvature measurement on loose-tube optical cable delivery length // Proceedings of SPIE. 2016. V. 9807. P. 98071A.

34. Бурдин В.А., Бурдин А.В. Рефлектометрические методы измерений распределений избыточной длины оптических волокон в модульных трубках кабеля // Фотоника. 2017. № 4 (64). С. 96-105.

35. Бурдин В.А. Способ измерения избыточной длины оптического волокна в модуле оптического кабеля // Патент RU2685066. 2019. Бюл. № 11.

36. Lundergan M.L., Zimmerman B.D., Waterman B. Mechanical and Optical Functionality of Field Aged Optical Ground Wire Cable // Proceedings of the National Fiber Optic Engineers Conference. 1996. P. 449-458.

37. Lundergan M.L., Dallas K.M. Field aging study shows strength of optical ground wire cable // Lightwave. 1997. V. 14. No. 11. P. 1-4.

38. Оптический кабель для любых сфер применения: каталог Инкаб, 2017. 184 c. https://lantrade.ru/upload/iblock/8a3/inkab_katalog-2017. $\operatorname{pdf}(11.04 .20)$.

39. РД 153-34.0-48.518-98. Правила проектирования, строительства и эксплуатации волоконно-оптических линий связи на воздушных линиях электропередачи напряжением 110 кВ и выше. - М., 1999. 109 с.

40. СО 153-34.48.519-2002. Правила проектирования, строительства и эксплуатации волоконно-оптических линий связи на воздушных линиях электропередачи напряжением 0,435 кB. - М., 2004. 83 c.

41. Монтаж проводов, грозозащитных тросов и волоконно-оптического кабеля связи под тяжением на ВЛ 220-750 кВ. Том 1. Монтаж проводов и грозозащитных тросов на ВЛ 220 кВ. - М.: ЗАО "Институт "Оргэнергострой", 2005. 42 с. 